\title{
Novel tools and challenges for jet physics in heavy-ion collisions at the LHC
}

\author{
Laura Havener* \\ Yale University \\ E-mail: laura.havener@yale.edu
}

\begin{abstract}
Jet measurements in heavy-ion collisions have a unique challenge due to the large fluctuating background from the underlying event. Recent advances in background subtraction techniques will be discussed that have allowed inclusive jet measurements to be made to lower jet transverse momentum and larger jet resolution parameters than ever before at the LHC. Additionally, it can be challenging to find observables that are sensitive to the particular in-medium effects in which we are interested in order to study the quark-gluon plasma. Recent applications of novel jet substructure tools for heavy-ion collisions will be discussed that are shown to be sensitive to jet quenching effects along with improvements in background subtraction techniques for these jet substructure measurements. These new tools allow jet measurements in heavy-ion collisions at the LHC to be unfolded for detector and background effects such that they can be compared to various theoretical predictions to help us better understand jet quenching.
\end{abstract}

The Eighth Annual Conference on Large Hadron Collider Physics-LHCP2020

25-30 May, 2020

online

${ }^{*}$ Speaker. 


\section{Introduction}

Jets are used as a probe of the quark-gluon plasma (QGP) produced in heavy-ion collisions (HICs). As jets move through the medium they interact leading to jet energy loss and substructure modification, a phenomena called jet quenching $[1,2,3]$. Jet quenching is interesting to study over a large range of scales, including the jet transverse momentum $p_{\mathrm{T}}$ and resolution parameter $R$, where in-medium effects are expected to vary $[4,5,6,7]$. Measuring jets in HICs can be challenging because of the large background from the underlying event (UE) of flowing particles underneath the jet that produces fluctuations on the order of the jet $p_{\mathrm{T}}$ itself [8]. Upward fluctuations in the UE can be taken as jets, called fakes, which sometimes prevent unfolding especially at lower jet $p_{\mathrm{T}}$ and larger $R$, regions of the phase space where the physics is of interest. Additionally, it can be challenging to find observables that are sensitive to in-medium effects, especially those that can be compared to first principle calculations to help constrain models.

In this proceeding, recent progress at the LHC in addressing these challenges will be discussed. For dealing with the large background new background subtraction techniques have been developed, which include machine learning techniques. In addition, new jet substructure tools are being utilized to isolate in-medium effects and constrain models to better understand the QGP.

\section{Overcoming the heavy-ion background}

Jet measurements in HICs at the LHC already cover a large jet $p_{\mathrm{T}}$ range, which can be illustrated using the nuclear modification factor $R_{\mathrm{AA}}$. Since jets are expected to lose energy in the QGP, the jet yield should be suppressed at a fixed value of $p_{\mathrm{T}}$ in HI compared to pp collisions where no medium is produced. This suppression is quantified using the $R_{\mathrm{AA}}$, which compares the yield in HICs to the cross section in pp collisions with a scale factor to account for the nuclear geometry. The ATLAS and CMS experiments focus on measuring jets at very high $p_{\mathrm{T}}$ with high precision $[9,10,11]$, where for example ATLAS measured inclusive jet suppression out to a TeV in jet $p_{\mathrm{T}}$ [9]. The ALICE experiment focuses on measuring jets at lower $p_{\mathrm{T}}$ taking advantage of precise tracking in the TPC, where inclusive jet suppression was measured over a range of 60-140 $\mathrm{GeV} / c$ [12]. Therefore, we see jet suppression over a large range in jet $p_{\mathrm{T}}$ but we would like to push these limits, particularly to lower jet $p_{\mathrm{T}}$ and larger $R$.

\subsubsection{ALICE machine learning technique}

A new method of background removal has been developed using a ML approach [13]. Recently ALICE implemented this method to measure inclusive jets to lower $p_{\mathrm{T}}$. The standard areabased (AB) method [8] used by ALICE effectively subtracts the average background but residual fluctuations remain large, leading to a large fake contribution. Previously when measuring inclusive jets, a leading track $p_{\mathrm{T}}$ cut was applied to remove fakes, which introduced a bias [12]. This new method uses ML techniques to correct the jet $p_{\mathrm{T}}$ by learning the difference between signal PYTHIA [14] jets and the background from properties of the jet including its constituents. This leads to a decrease in residual fluctuations, but introduces a fragmentation bias because PYTHIA jets in pp collisions have different fragmentation patterns than quenched jets in HICs $[15,16]$. 
In ALICE, this new method has been applied to both charged particle and full jets, which contain charged tracks and neutral clusters $[17,18]$. The performance of this method for full jets is shown in left panel of Figure 1 compared to the $\mathrm{AB}$ method. The performance is evaluated using the $\delta p_{\mathrm{T}}=p_{\mathrm{T}, \mathrm{rec}}-p_{\mathrm{T}, \mathrm{det}}$, where $p_{\mathrm{T}, \text { rec }}$ is the ML corrected $p_{\mathrm{T}}$, and $p_{\mathrm{T}, \mathrm{det}}$ is the PYTHIA detector level jet $p_{\mathrm{T}}$, which is narrower for smaller residual fluctuations. The ML method shows a significant improvement over the $\mathrm{AB}$ method, allowing us to unfold to lower jet $p_{\mathrm{T}}$. This method has been applied to ALICE data in Ref. [18] and the fragmentation bias has been quantified, allowing full jets to be measured down to $40 \mathrm{GeV} / c$ with reduced systematic uncertainties.

\subsubsection{CMS large- $R$ jets}

CMS has the ability to measure jets at very high $p_{\mathrm{T}}$ where the background effects are smaller. The jet-by-jet constituent subtraction method [19] that was originally developed for pp collisions to remove pile-up helps improve the jet energy resolution (JER) when used for HI jets in CMS [11]. The method works by estimating the background energy density inside each jet (with an UE modulation from flow) and adding infinitesimal small "ghosts" to the jets whose transverse momentum is negative. The ghosts and particles are then combined based on how close they are to each other, then particles and ghosts with less than zero total momentum are removed from the jet. The right panel of Figure 1 shows the JER for jets with different $R$. The JER is shown to improve substantially with increasing jet $p_{\mathrm{T}}$, especially for larger $R=1.0$ jets. This allowed CMS to measure the $R_{\mathrm{AA}}$ for $R=1.0$ jets for the first time in HICs in Ref. [11].
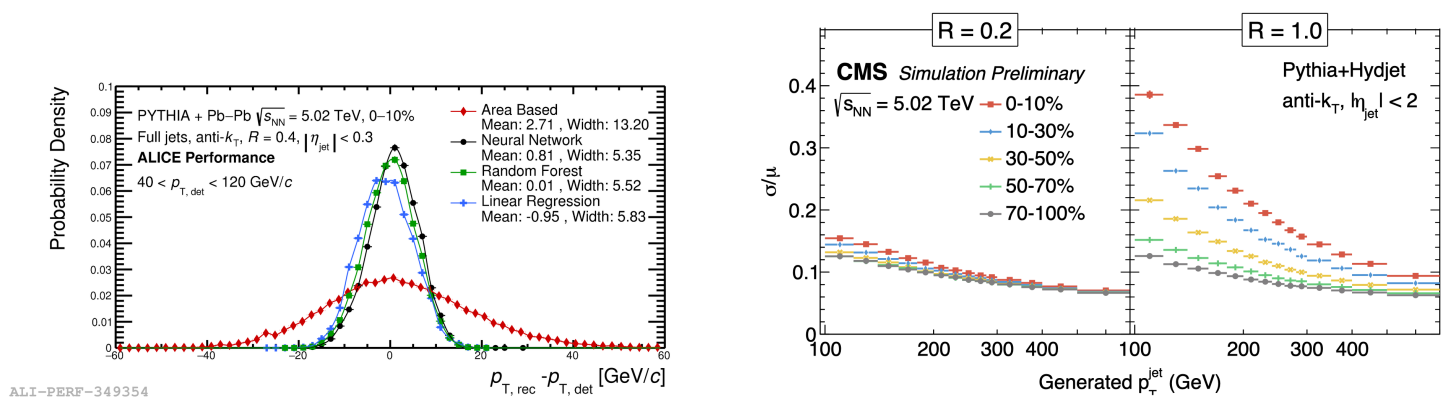

Figure 1: Left: The $\delta p_{\mathrm{T}}=p_{\mathrm{T}, \text { rec }}-p_{\mathrm{T}, \mathrm{det}}$ distributions for $R=0.4$ jets in central collisions (0-10\%) with ALICE [18]. Right: The JER distributions for $R=0.2$ and $R=1.0$ central jets in CMS [11].

\section{Novel jet substructure tools}

There are many examples of jet substructure measurements at the LHC. This includes the jet mass which appears insensitive to medium modification possibly due to a cancellation of inmedium effects $[20,21,22]$. The high energy physics community has developed tools in pp collisions to access hard splittings inside a jet by removing the soft background contribution. Recently, these tools were found to be useful when applied to HICs because they help to remove the soft background and some of the soft signal, which can separate out medium effects that might cancel in other observables. These variables are also directly calculable in $\mathrm{pQCD}$, allowing for comparisons to theoretical predictions. ALICE uses Soft Drop (SD) [23] grooming where jets are reclustered 
with the Cambridge-Aachen (C/A) [24] algorithm and a condition is placed on the shared momentum fraction between the splittings. ATLAS reclusters smaller $R$ jets into larger $R$ jets using the anti- $k_{\mathrm{T}}$ algorithm [24] with a cut on the smaller $R$ jet $p_{\mathrm{T}}$.

Jet splitting measurements in HICs are challenging because background particles can be picked up as a subjet in a splitting over the real subjet. This leads to incorrect splittings that cause large offdiagonal contributions in the response matrix, making unfolding impossible. The LHC experiments have come up with solutions to this by measuring in regions of phase space where the background is suppressed. ALICE uses smaller $R=0.2$ jets in central $(0-10 \%)$ and larger $R=0.4$ jets in semi-central (30-50\%) collisions for charged-particle jets between $60-80 \mathrm{GeV} / c$ [25]. ATLAS uses higher $p_{\mathrm{T}}>250 \mathrm{GeV} / c R=1.0$ jets from clustered $R=0.2$ jets [26]. Both experiments additionally require more symmetric splittings to further suppress the background, with ALICE using a stronger grooming condition and ATLAS restricting the $R=0.2$ jets to have $p_{\mathrm{T}}>35 \mathrm{GeV} / c$.

In addition to the phase space restrictions, ALICE uses the event-by-event constituent subtraction method instead of the jet-by-jet (described in Section 2.0.2) [19,27]. The left panel of Figure 2 shows the groomed jet radius $R_{\mathrm{g}}$ distribution for PYTHIA jets embedded in real $\mathrm{Pb}-\mathrm{Pb}$ data, where a secondary peak from the background splittings at large values is seen [25]. The event-by-event method is shown to reduce this contribution significantly. The center panel of Figure 2 also shows that the peak is again significantly reduced when a stronger grooming condition is used $\left(z_{\text {cut }}=0.1\right.$ to 0.2). In ATLAS, combining the smaller $R=0.2$ jets into larger $R=1.0$ jets allows these measurements to take advantage of the precise background subtraction for smaller $R=0.2$ jets [26]. The JER for these reclustered $R=1.0$ jets is shown in the right panel of Figure 2, where it is only $10 \%$ for jets above $200 \mathrm{GeV} / c$ in central collisions. These techniques are used to measure the unfolded $R_{\mathrm{g}}$ in ALICE and the unfolded $k_{\mathrm{T}}$ splitting scaling in ATLAS as discussed in Refs. [25] and [26], respectively. Both are the first measurements of their kind and demonstrate a significant modification in HICs, successfully isolating in-medium effects.
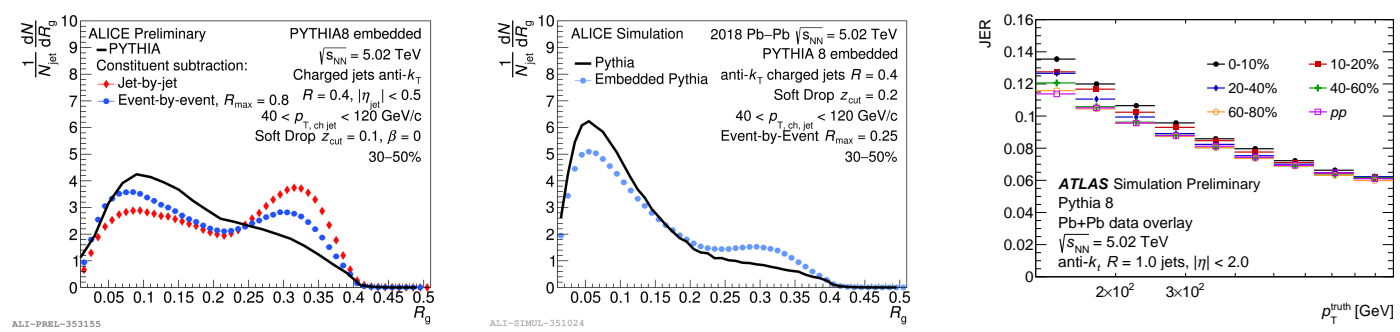

Figure 2: Left: The $R_{\mathrm{g}}$ distribution $\left(z_{\mathrm{cut}}=0.1\right)$ for embedded PYTHIA and PYTHIA for the event-by-event and jet-by-jet subtraction method in ALICE [25]. Center: The embedded $R_{\mathrm{g}}$ distributions for $z_{\text {cut }}=0.2$. Right: The JER for the reclustered $R=1.0$ jets for different centralities in ATLAS [26].

\section{Conclusion}

New background subtraction techniques at the LHC have allow for jet measurements at lower $p_{\mathrm{T}}$ and larger $R$ than ever before. Additionally, jet substructure tools applied to HICs seem to be sensitive to jet quenching effects. In general, improving jet measurement tools allow for direct comparison of unfolded results to theoretical calculations to help constrain jet quenching models. 


\section{References}

[1] G.-Y. Qin and X.-N. Wang, Jet quenching in high-energy heavy-ion collisions, Int. J. Mod. Phys. E 24 (2015) 1530014.

[2] J.-P. Blaizot and Y. Mehtar-Tani, Jet structure in heavy ion collisions, Int. J. Mod. Phys. E 24 (2015) 1530012.

[3] A. Majumder and M. van Leeuwen, The theory and phenomenology of perturbative QCD based jet quenching, Prog. Part. Nucl. Phys. 66 (2011) 41.

[4] D. Pablos, Jet Suppression From a Small to Intermediate to Large Radius, Phys. Rev. Lett. 124 (2020) $052301[1907.12301]$.

[5] R. Kunnawalkam Elayavalli and K.C. Zapp, Medium response in JEWEL and its impact on jet shape observables in heavy ion collisions, JHEP 07 (2017) 141 [1707. 01539].

[6] H.T. Li and I. Vitev, Inclusive heavy flavor jet production with semi-inclusive jet functions: from proton to heavy-ion collisions, JHEP 07 (2019) 148 [1811.07905].

[7] Y. He, S. Cao, W. Chen, T. Luo, L.-G. Pang and X.-N. Wang, Interplaying mechanisms behind single inclusive jet suppression in heavy-ion collisions, Phys. Rev. C 99 (2019) 054911 [1809.02525].

[8] ALICE collaboration, Measurement of Event Background Fluctuations for Charged Particle Jet Reconstruction in Pb-Pb collisions at $\sqrt{s_{N N}}=2.76 \mathrm{TeV}, \mathrm{JHEP} 03$ (2012) 053 [1201.2423].

[9] ATLAS collaboration, Measurement of the nuclear modification factor for inclusive jets in $\mathrm{Pb}+\mathrm{Pb}$ collisions at $\sqrt{s_{\mathrm{NN}}}=5.02 \mathrm{TeV}$ with the ATLAS detector, Phys. Lett. B 790 (2019) 108.

[10] CMS collaboration, Measurement of inclusive jet cross sections in and collisions at $=2.76 \mathrm{TeV}$, Phys. Rev. C 96 (2017) 015202.

[11] ATLAS collaboration, Measurement of the nuclear modification factor for inclusive jets in $\mathrm{Pb}+\mathrm{Pb}$ collisions at $\sqrt{s_{\mathrm{NN}}}=5.02$ TeV with the ATLAS detector, Phys. Lett. B 790 (2019) 108 [1805.05635].

[12] ALICE collaboration, Measurements of inclusive jet spectra in pp and central pb-pb collisions at $\sqrt{s_{N N}}=5.02 \mathrm{TeV}$, Phys. Rev. C 101 (2020) 034911.

[13] R. Haake and C. Loizides, Machine Learning based jet momentum reconstruction in heavy-ion collisions, Phys. Rev. C 99 (2019) 064904 [1810.06324].

[14] T. Sjostrand, S. Ask, J.R. Christiansen, R. Corke, N. Desai, P. Ilten et al., An introduction to PYTHIA 8.2, Comput. Phys. Commun. 191 (2015) 159.

[15] CMS collaboration, Measurement of Jet Fragmentation in PbPb and pp Collisions at $\sqrt{s_{N N}}=2.76$ TeV, Phys. Rev. C 90 (2014) 024908 [1 406 . 0932].

[16] ATLAS collaboration, Measurement of jet fragmentation in $\mathrm{Pb}+\mathrm{Pb}$ and $\mathrm{pp}$ collisions at $\sqrt{s_{N N}}=5.02 \mathrm{TeV}$ with the ATLAS detector, Phys. Rev. C 98 (2018) 024908 [1805. 05424 ].

[17] ALICE collaboration, Machine Learning based jet momentum reconstruction in Pb-Pb collisions measured with the ALICE detector, in 2019 European Physical Society Conference on High Energy Physics, 9, 2019 [1909.01639].

[18] ALICE collaboration, Inclusive Jet Measurements in Pb-Pb Collisions at $5.02 \mathrm{TeV}$ with ALICE using Machine Learning Techniques, in 10th International Conference on Hard and Electromagnetic Probes of High-Energy Nuclear Collisions: Hard Probes 2020 , 9, 2020 [2009. 022 69]. 
[19] P. Berta, M. Spousta, D.W. Miller and R. Leitner, Particle-level pileup subtraction for jets and jet shapes, JHEP 06 (2014) 092.

[20] ALICE collaboration, First measurement of jet mass in Pband pcollisions at the LHC, Phys. Lett. B 776 (2018) 249 [1702.00804].

[21] CMS collaboration, Measurement of the groomed jet mass in PbPb and pp collisions at $\sqrt{s_{\mathrm{NN}}}=5.02$ TeV, JHEP 10 (2018) 161 [1805.05145].

[22] ATLAS collaboration, Measurement of $R=0.4$ jet mass in $P b+P b$ and pp collisions at $\sqrt{s_{\mathrm{NN}}}=5.02$ TeV with the ATLAS detector, .

[23] A.J. Larkoski, S. Marzani, G. Soyez and J. Thaler, Soft Drop, JHEP 05 (2014) 146.

[24] Y.L. Dokshitzer, G. Leder, S. Moretti and B. Webber, Better jet clustering algorithms, JHEP 08 (1997) 001 [hep-ph/9707323].

[25] A. Collaboration, Physics Preliminary Summary: Measurements of the groomed jet radius and groomed momentum fraction in pp and Pb-Pb collisions at $\sqrt{s_{\mathrm{NN}}}=5.02 \mathrm{TeV}, \mathrm{CDS} C E R N$ (2020).

[26] ATLAS collaboration, Measurement of suppression of large-radius jets and its dependence on substructure in $\mathrm{Pb}+\mathrm{Pb}$ at $5.02 \mathrm{TeV}$ by ATLAS detector, .

[27] P. Berta, L. Masetti, D. Miller and M. Spousta, Pileup and Underlying Event Mitigation with Iterative Constituent Subtraction, JHEP 08 (2019) 175. 